\title{
Effective Spatial Characterization System Using Density-Based Clustering
}

\author{
Chan-Min Ahn ${ }^{1}$, Jae-Hyun You ${ }^{2}$, Ju-Hong Lee ${ }^{3, *}$, and Deok-Hwan Kim ${ }^{4}$ \\ ${ }^{1,2,3}$ Department of Computer science and Engineering, Inha-University, Korea \\ ${ }^{4}$ Department of Electronic and Engineering, Inha-University, Korea \\ ${ }^{1,2}$ \{ahnch1, you\} edatamining.inha.ac.kr, \\ ${ }^{3,4}$ \{juhong, deokhwan\}@inha.ac.kr
}

\begin{abstract}
Previous spatial characterization methods does not analyze well spatial regions for a given query since it only focus on characterization for user's pre-selected area and without consideration of spatial density. Consequently, the effectiveness of characterization knowledge is decreased in these methods. In this paper, we propose a new hybrid spatial characterization system combining the density-based clustering module which consists of the attribute removal generalization and the concept hierarchy generalization. The proposed method can generate characteristic rule and apply density-based clustering to enhance the effectiveness of generated rules.
\end{abstract}

\section{Introduction}

Recently, the need for the spatial data mining has increased in many applications such as geographic information system, weather forecasting, and market analysis, etc. The study on spatial data mining techniques is as follows: the spatial characteristic rule to extract summary information of spatial and non-spatial data, spatial association rule to find the spatial relationship between data, and the spatial clustering to partition spatial data objects into a set of meaningful subclasses, and so on $[6,7,8,9]$.

Especially, spatial characterization is one of methods for discovering knowledge by aggregating non-spatial data and spatial data [4]. Many previous data mining systems support spatial characterization methods. Economic Geography and GeoMiner are the representative spatial data mining system which support spatial knowledge discovery [7, 9].

Ester et al. suggest Economic Geography system based on BAVIRIA database [9]. Economic Geography uses a part of entire database and performs characterization by using the relative frequency of spatial and non-spatial data. That is, it increases effectiveness of characterization using only relative frequencies of objects nearest to target object. GeoMiner system enhances DBMiner system to treat both spatial and nonspatial data [7]. This system enables knowledge discovery for association, clustering, characterization, and classification. It also presents both NSD(Non-Spatial Data

\footnotetext{
* Corresponding author.
} 
Dominant Generalization) and SD(Spatial data Dominant Generalization) algorithms for spatial characterization. GeoMiner has the advantage of presenting the appropriate type of discovered knowledge according to the user's needs.

The previous characterization systems have the problems as follows: first, their characterization methods do not analyze well spatial regions for a given user query since they only use regions predefined by domain experts. Second, the user should describe the query range directly to reduce the search scope $[6,8]$.

In this paper, to solve the previous problems, we propose an effective spatial characterization method combining spatial data mining module which extends generalization technique and density-based clustering module. The density-based clustering module enables the system to analyze the spatial region and to reduce the search scope for a given user query. Our characterization method can generate useful characteristic rule and apply the density-based clustering to enhance the effectiveness of generated rules. Its effectiveness is measured by information gain which uses the entropy of selected data set [1].

The rest of the paper is organized as follows: in Section 2, we describe the proposed spatial characterization method using density-based clustering. In Section 3, we introduce a new characterization system implementing the suggested method. Section 4 contains the result of our experiment. Finally, Section 5 summarizes our works.

\section{Spatial Characterization Method Using Density-Based Clustering}

In this section, we describe a spatial characterization method using density-based clustering. Our characterization method first performs the density-based clustering on spatial data and then extracts summary information by aggregating non-spatial data and spatial data with respect to each cluster.

\subsection{Proposed Spatial Characterization Method}

Spatial characterization extracts the summary information of data class with respect to the search scope from the given spatial data and non-spatial data. The proposed spatial characterization method consists of five steps shown in Fig.1.

The first step is to retrieve task-relevant data in the search scope from spatial database. Task-relevance data are spatial data and non-spatial data of database tuples related with the user query. After that, we perform density based clustering with respect to spatial data of retrieved task-relevant data. Note that this step is described separately in Section 2.2. As a result of clustering, the search scope is reduced to the objects within the selected spatial clusters. In the third and forth step, for the non-spatial attribute of each object in the spatial clusters, generalization is performed until the specific user threshold is satisfied. The proposed generalization module consists of an attribute removal generalization and a concept hierarchy generalization. 


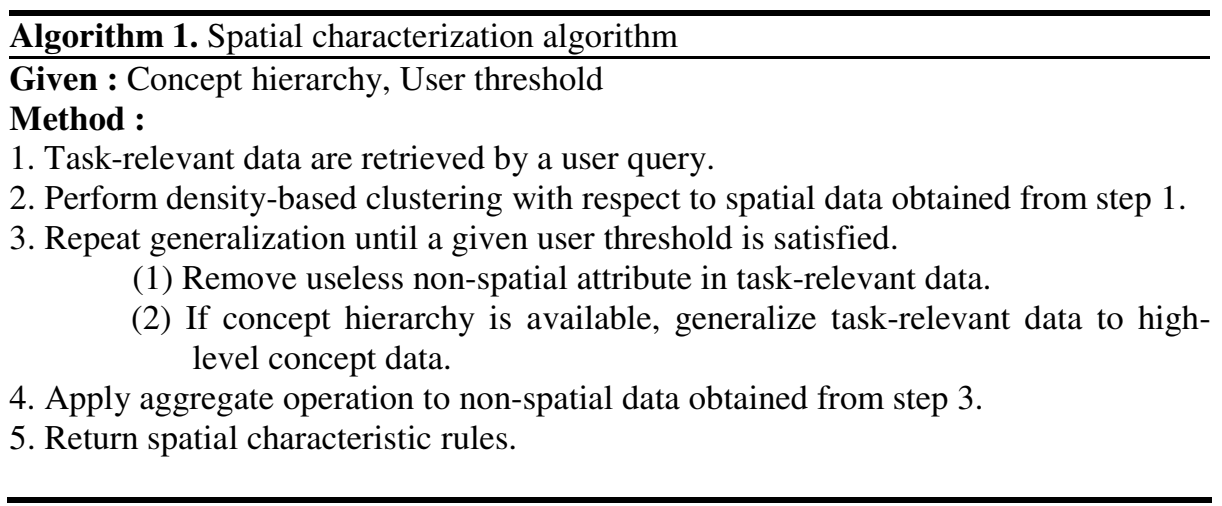

Fig. 1. Spatial characterization algorithm

The attribute removal generalization is used in the process of converting nonspatial task-relevant data into summary information. The attributes are removed in the following cases: (1) tuple values with respect to a specific attribute are all distinct or the attribute has more distinct tuple values than the user threshold. (2) The tuple value with respect to a specific attribute cannot be replaced with high level concept data.

After attribute removal steps, non-spatial task-relevant data is converted to summary information using concept hierarchy. A concept hierarchy is a sequence of mappings from a set of low-level raw data to more general high-level concepts which represent summary information [5]. At the end, the algorithm returns spatial characteristic rules.

This generalization algorithm is the key point of the proposed spatial characterization. Extracting generalized knowledge from spatial database using spatial characterization requires generalization of spatial data and non-spatial data. Thus, the generalization module performs the generalization task for non-spatial task-relevant data by using the user defined concept hierarchy.

\subsection{Density-Based Spatial Clustering}

Spatial data mining uses both non-spatial data and spatial data. A tuple in the database includes a spatial object. Spatial object has the coordinate information of spatial data related with the non-spatial data. Spatial clustering is used to partition the areas the user wants to search $[2,3,9,10]$. We use DBSCAN as the density-based spatial clustering module to group spatial areas for spatial characterization since it can be used as a basic tool for spatial analysis $[9,10]$.

DBSCAN merges regions with sufficient high density into clusters. The proposed method supports not only a point type but also a polygon type. We extend DBSCAN with minmax distance function to support two types of spatial data. Let object be a point data or a polygon data. In order for objects to be grouped, there must be at least a minimum number of objects called MinObj in $\varepsilon$-neighbourhood, $\mathrm{Ne}(\mathrm{p})$, from an 
object $p$, given a radius $\varepsilon$ where $p$ and $q$ are objects and $D$ is a data set. If $p$ and $q$ are point type objects, their distance can be calculated by Euclidian distance function [9]. Otherwise, their distance can be calculated by minmax distance function [11]. The minmax distance function is to compute the minimum value of all the maximum distances between objects.

$$
N e(p)=\{q \in D \mid \operatorname{dist}(p, q) \leq \varepsilon\}
$$

Fig. 2 illustrates clustering example of polygon type objects with respect to the map of the province in Incheon city. To cluster polygon type objects, we calculate MBR of each polygon object. After that, we calculate the minmax distance values between the center of selected MBR and other MBRs. The remaining step is the same as the clustering process of the point type objects.



Fig. 2. Clustering example of polygon type object

Fig. 3 illustrates the extended DBSCAN algorithm. For this clustering algorithm, we need some parameters. Let SetOfbject be a set of spatial data, NoOfClusters be the given number of clusters, and $r$ be a radius of $\varepsilon$-neighborhood.

The complexity of the expended DBSCAN algorithm can be expressed by $O(n 2)$, where $\mathrm{n}$ is the number of objects.

\section{Design and Implementation of Spatial Characterization System}

In this section, we describe each component of the proposed spatial characterization system and an application example.

The proposed spatial characterization system consists of spatial data mining query processor, spatial data mining module, spatial database, and spatial clustering module. We use a SMQL query language for spatial data mining. SMQL is a spatial data mining language used in GMS spatial database system [13].

Example 1 shows an SMQL query of an application for the proposed system. Taskrelevant data in Table.1 are intermediate query results using Example 1. 


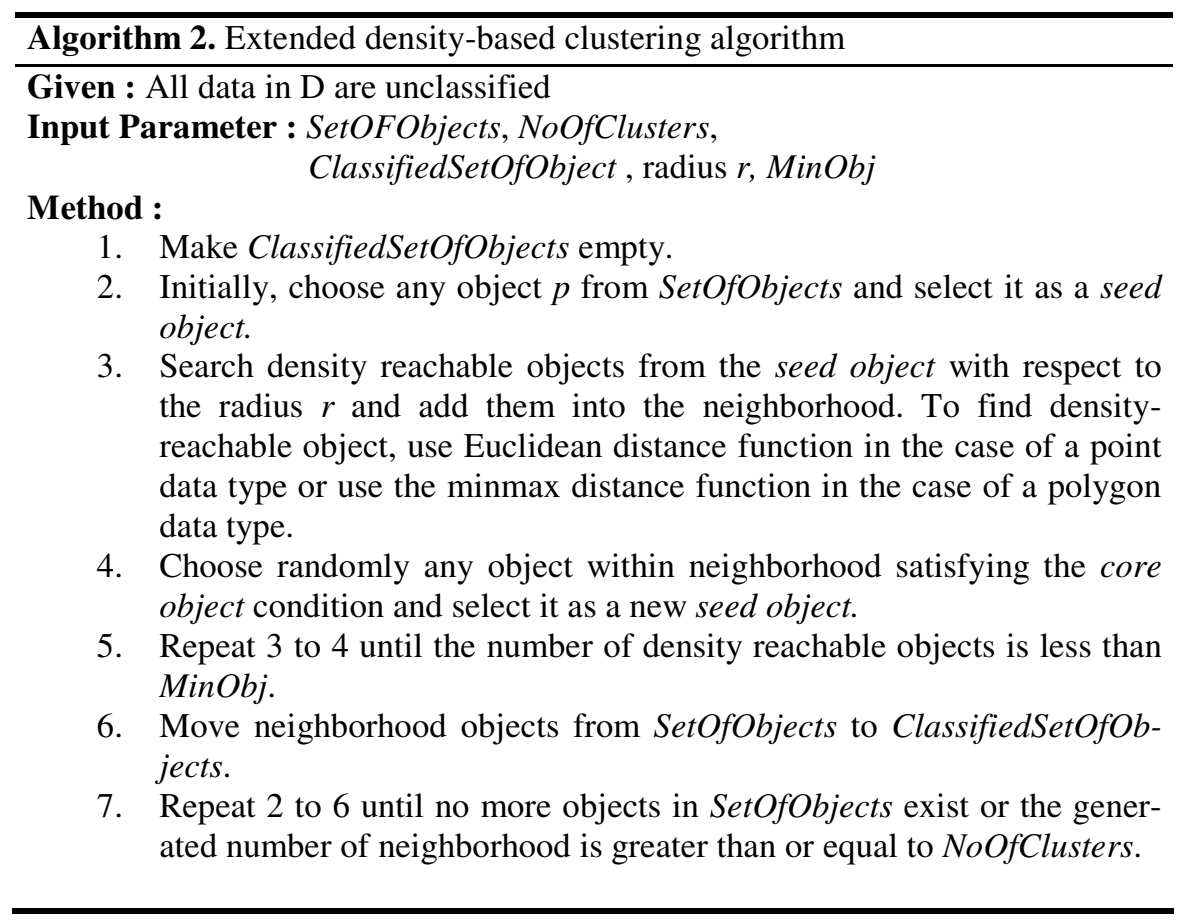

Fig. 3. Extended density-based clustering algorithm

Example 1. SMQL query for characterization with respect to annual_income, education, and age of women who reside in Incheon city.

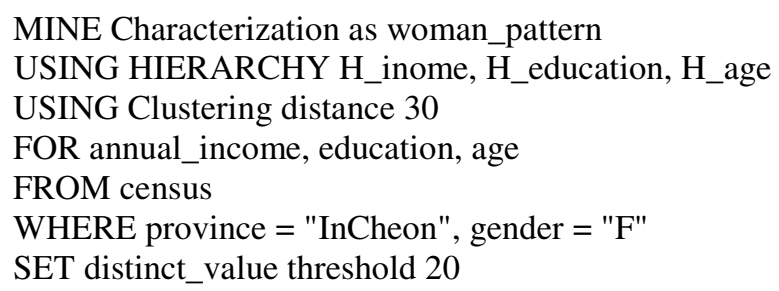

[Step1] Given a SMQL query statement in Example.1, the system collects annual_income, education, age, and coordinate information attribute values of taskrelevant data from census table in the spatial database. Please refer to [13] for SMQL query processor.

[Step 2] Clustering is performed by using coordinate information, which is stored in object pointer attribute, with respect to spatial task-relevant data.

[Step3] In this step, low level attribute values are replaced with matching high level attribute value in the concept hierarchy. The system generalizes task-relevant data in Table.1 with respect to annual_income attribute. This generalization will make the high opportunity of aggregation task. 
[Step4] Characterization that aggregates all tuples is performed. Table 2 shows the aggregated data as the result of SMQL query shown in Example 1.

Table 1. Task-relevant data

\begin{tabular}{cccccc}
\hline id & Annual_income & age & education & object & $\ldots$ \\
\hline 1 & 2580 & 45 & Univ & $<\mathrm{X}, \mathrm{Y}\rangle$ & $\ldots$ \\
2 & 2400 & 40 & Senior & $<\mathrm{X}, \mathrm{Y}\rangle$ & $\ldots$ \\
3 & 3850 & 36 & Master & $<\mathrm{X}, \mathrm{Y}\rangle$ & $\ldots$ \\
4 & 1780 & 27 & Univ & $<\mathrm{X}, \mathrm{Y}\rangle$ & $\ldots$ \\
$\ldots$ & $\ldots$ & $\ldots$ & $\ldots$ & $\ldots$ & $\ldots$ \\
\hline
\end{tabular}

Table 2. A result of spatial characterization

\begin{tabular}{ccccc}
\hline Cluster & annual_income & Age & education & count \\
\hline C1 & Middle & middle & Higher & 481 \\
C3 & Middle & middle & Secondary & 316 \\
C1 & Middel high & middle & Higher & 156 \\
$\ldots$ & $\ldots$ & $\ldots$ & $\ldots$ & $\ldots$ \\
\hline
\end{tabular}

\section{Evaluation and Result}

We use a real map in the province of Incheon city. The data have 8000 objects which consisting of points and polygons. We perform an experiment and evaluate the result using entropy.

Entropy $[1,12]$ is used to measure the purity of target data in information theory. When the data set $S$ is classified into $c$ subsets, the entropy of $S$ is defined as follows:

$$
\operatorname{Entropy}(S)=-\sum_{i=1}^{c} p_{i} \log _{2} p_{i}
$$

where $S$ is a selected data set, $p_{i}$ is the ratio that the set $S$ includes class $i$, and $C$ is the number of set that is distinct from the set $S$.

The average weight of an attributes is defined as:

$$
W=w_{n i} / T
$$

where $w_{n i}$ is the weight of $n i, n i$ represents randomly selected attribute, and $T$ is the total weight.

In order to measure whether the result of spatial characterization reflects well data distribution in the database, the entropy and weight of attribute is used. Thus, the information gain from the result of spatial characterization using the entropy and the weight of each attribute is defined as follows:

$$
\operatorname{Gain}(G)=E-W_{a} E_{a}+W_{b} E_{b}
$$


where $E$ is a total entropy, $a$ is a data set, $b$ is another data set distinct from $a, W_{a}$ is an average weight of $a, W_{b}$ is an average weight of $b, E_{a}$ is an entropy of $a$, and $E_{b}$ is an entropy of $b$, respectively.

We apply the information gain to the sample data used in application example 1 mentioned in section 3. Characterization without clustering denotes the previous method [7,9] using only data generalization while characterization with clustering denotes the proposed method. Fig 4 shows the experimental result that compares characterization with clustering and characterization without clustering in terms of the information gain.

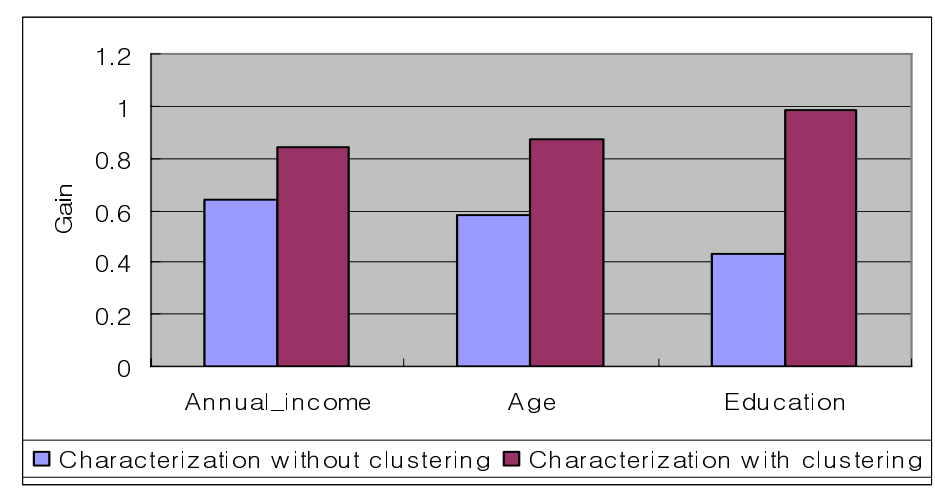

Fig. 4. Result of two spatial characterization using information gain

The experimental result demonstrates that the characterization with clustering is more effective than that without clustering with respect to annual income, age, and education attributes, respectively.

\section{Conclusion}

We propose a new spatial characterization method that generalizes spatial objects and groups them by using DBSCAN density-based clustering as automatically selected areas, and aggregates them on non-spatial attributes. The proposed spatial characterization method has the properties as follows: first, we use a density based clustering to automatically select search scope. Second, the method can eliminate unnecessary spatial objects using attribute removal and concept hierarchy generalization operation.

The elimination of unnecessary spatial objects can give us useful knowledge, where information gain is high. The experimental result demonstrates that the performance of the proposed characterization method is better than that of the previous characterization method. 


\section{Acknowledgement}

This research was supported by the MIC(Ministry of Information and Communication), Korea, under the ITRC(Information Technology Research Center) support program supervised by the IITA(Institute of Information Technology Assessment).

\section{References}

1. Baeza-Yaters, R., Ribeiro-Neto, B.: Modern Information Retrieval. ACM Press (2000) pp.144-149

2. Edwin, M., Knorr, Raymond, Ng.: Robust Space Transformations for Distance-based Operations. SIGKDD, San Francisco, California USA 2 (2001) pp.126-135

3. E., Shaffer, and M., Garland.: Efficient Adaptive Simplification of Massive Meshes. In Proceedings of IEEE Visualization 2001 (2001) pp.127-134

4. J., Amores, and P., Radeva.: Non-Rigid Registration of Vessel Structures in IVUS Images, Iberian Conference on Pattern Recognition and Image Analysis. Puerto de Andratx, Mallorca, Spain, Lecture Notes in Computer Science, Springer-Verlag 2652 (2003) pp.45-52

5. J., Han, and M., Kamber.: Data Mining Concept and Techniques. Morgan Kaufman (2001) pp.130-140

6. J., Han, Y., Cai, and N., Cercone.: Knowledge Discovery in Databases : An AttributeOriented Approach. Proceedings of the $18^{\text {th }}$ VLDB Conference. Vancouver, British Columbia, Canada (1992) pp.547-559

7. J., Han, K., Koperski, and N., Stefanovic.: GeoMiner : A system prototype for spatial data mining, Proceedings of 1997 ACM-SIGMOD International Conference on Management of Data 262 (1997) pp.553-556

8. M., Ester, H., -P., Kriegel, and J., Sander.: Knowledge discovery in large spatial databases : Focusing Techniques for Efficient Class Identification. In proc. $4^{\text {th }}$ Intl. Symp. on Large Spatial Databases 951 (1995) pp.67-82

9. M., Ester, H., -P., Kriegel, and J., Sander.: Algorithms and applications for spatial data mining. Geographic Data Mining and Knowledge Discovery, London: Taylor and Francis (2001) pp.160-187

10. M., Ester, H., -P., Kriegel, J., Sander, and X., Xu.: A Density-Based Algorithm for Discovering Clusters in Large Spatial Databases with Noise. In Proc. of ACM SIGMOD $3^{\text {rd }}$ International Conference on Knowledge Discovery and Data Mining. AAAI Press (1996) pp.226-231

11. N., Roussopoulos, S., Kelley, and F., Vincent.: Nearest Neighbor Queries. In Proc. of ACM SIGMOD Intl. Conf. on Management of Data, San Jose, CA (1995) pp.71-79

12. N., Roy, and C., Earnest.: Dynamic Action Space for Information Gain Maximization in Search and Exploration. In Proc. of American Control Conference, Minneapolis 12 (2006) pp.1631-1636

13. S., Park, S.H., Park, C.M., Ahn, Y.S., Lee, J.H., Lee,: Spatial Data Mining Query Language for SIMS. Spring conference of The Korea Information Science Society 311 (2003) pp.70-72 